\title{
Two-dimensional solitons with hidden and explicit vorticity in bimodal cubic-quintic media
}

\author{
A. S. Desyatnikov, ${ }^{1}$ D. Mihalache, ${ }^{2,3}$ D. Mazilu, ${ }^{2,3}$ B. A. Malomed, ${ }^{4}$ C. Denz,${ }^{5}$ and F. Lederer ${ }^{3}$ \\ ${ }^{1}$ Nonlinear Physics Centre and Centre for Ultra-high bandwidth Devices for Optical Systems (CUDOS), Research School of Physical \\ Sciences and Engineering, Australian National University, Canberra ACT 0200, Australia \\ ${ }^{2}$ Department of Theoretical Physics, Institute of Atomic Physics, P.O. Box MG-6, Bucharest, Romania \\ ${ }^{3}$ Institute of Solid State Theory and Theoretical Optics, Friedrich-Schiller Universität Jena, Max-Wien-Platz, 1, D-07743 Jena, Germany \\ ${ }^{4}$ Department of Interdisciplinary Studies, School of Electrical Engineering, Faculty of Engineering, Tel Aviv University, \\ Tel Aviv 69978, Israel \\ ${ }^{5}$ Institute of Applied Physics, Westfälische Wilhelms-Universität Münster, D-48149 Münster, Germany
}

(Received 20 September 2004; published 28 February 2005)

\begin{abstract}
We demonstrate that two-dimensional two-component bright solitons of an annular shape, carrying vorticities $(m, \pm m)$ in the components, may be stable in media with the cubic-quintic nonlinearity, including the hidden-vorticity (HV) solitons of the type $(m,-m)$, whose net vorticity is zero. Stability regions for the vortices of both $(m, \pm m)$ types are identified for $m=1,2$, and 3, by dint of the calculation of stability eigenvalues, and in direct simulations. In addition to the well-known symmetry-breaking (external) instability, which splits the ring soliton into a set of fragments flying away in tangential directions, we report two new scenarios of the development of weak instabilities specific to the HV solitons. One features charge flipping, with the two components exchanging angular momentum and periodically reversing the sign of their spins. The composite soliton does not directly split in this case; therefore, we identify such instability as an intrinsic one. Eventually, the soliton splits, as weak radiation loss drives it across the border of the ordinary strong (external) instability. Another scenario proceeds through separation of the vortex cores in the two components, each individual core moving toward the outer edge of the annular soliton. After expulsion of the cores, there remains a zero-vorticity breather with persistent internal vibrations.
\end{abstract}

DOI: 10.1103/PhysRevE.71.026615

PACS number(s): 42.65.Tg, 42.65.Jx, 42.65.Sf

\section{INTRODUCTION}

Optical vortex solitons are two- or three-dimensional (2D or 3D) dark or bright self-trapped light beams with an embedded phase dislocation, which lends them an angular momentum ("spin"). As topologically nontrivial self-trapped states, the vortices have attracted a great deal of attention, which was additionally enhanced by a potential which the 2D ones may have as reconfigurable conduits for weak optical signals [1-3]. Vortex solitons of the bright type are of special interest in both respects. In particular, being relatively compact objects, they make it possible to realize sophisticated multivortex configurations. On the other hand, experimental creation of bright solitons is hampered by the fact that, in media with the simplest collapse-free nonlinearities (quadratic or saturable), they are subject to strong azimuthal instability, which splits them into a set of ordinary (zero-spin) solitons [4,5].

Nevertheless, as was first observed in direct simulations of a model with the cubic-quintic (CQ) nonlinearity in Ref. [6] and later investigated in detail by more accurate methods (see a review in Ref. [3]), bright vortices with topological charge $m=1$ [6], $m=2$ [3], and $m \geqslant 3$ [7] may be stable if the model features competing self-focusing and self-defocusing nonlinearities. Another example corroborating this conclusion is a model combining quadratic and self-defocusing cubic nonlinearities [8].

It is relevant to mention that patterns of a similar type, in the form of "optical necklaces"-i.e., ring-shaped chains of the fundamental solitons-were introduced in the model with Kerr (cubic) nonlinearity [9]. The necklaces may also be constructed with nonzero spin, including the case when it is noninteger. However, the necklaces are not stationary objects, as they spread out and eventually disintegrate. A possibility to (practically) stabilize necklacelike patterns is to introduce a vectorial interaction with a fundamental soliton, which helps to support vortex-, dipole-, and multipole-mode structures in another component [10]. The stabilization by vectorial interaction allows one to construct stationary necklace-ring vector solitons [11] with azimuthally modulated (necklace-type) components, whose densities sum up to an azimuthally uniform distribution of the total intensity. However, multipole and necklace-ring vector solitons are subject to an azimuthal instability, except for the dipolemode vector soliton [12] and vortex-mode soliton close to a bifurcation [13]. A particular class of such (generally, unstable) solutions is a vector vortex soliton with equal amplitude distributions in both components [14].

On the contrary to the above-mentioned diverse unstable configurations, stable two-component vortex solitons in the CQ model have been identified in Refs. [15,16]. A challenging issue is the possibility of the existence of stable vectorial solitons of the $(m,-m)$ type, which would feature the same annular shape (with a hole in the center) as the bright scalar or vectorial vortices with the spins, respectively, $m$ or $(m, m)$, but with zero net spin. The possibility of the existence of such objects is obvious if the coupling between the components is of the cross-phase modulation (XPM) type-i.e., insensitive to their relative phase- - then, there is no difference in the shape between stationary vortex solitons of the $(m, m)$ and $(m,-m)$ types. However, in the simplest collapse-free 
model with saturable nonlinearity, the $(m, \pm m)$ vector vortex solitons are unstable, although it was demonstrated that the instability may be partly suppressed for the $(m,-m)$ vortices $[14,17]$. A stabilizing effect of the incoherent interaction of counterrotating vortices was also demonstrated in an anisotropic photorefractive self-defocusing medium [18].

Compound vortices with the spin components $(1,-1,0)$ and $(1,1,2)$ were also studied in the three-wave model of the type-II second-harmonic generation, with two components of the fundamental wave and one second-harmonic component $[19,20]$. It was shown that, in this model per se, vortices of both types are unstable-against fusion into an ordinary zero-spin soliton or splitting, respectively. The addition of a stabilizing repulsive cubic interaction makes the life expectancy of the vortices much longer, but no case of complete stabilization has been found [20].

In this work, we demonstrate that, in contrast with the previously studied models, the $(m,-m)$ vortex solitons are rigorously stable in a certain parameter region in the $\mathrm{CQ}$ model. In fact, this result opens up a new class of stable 2D solitons with hidden vorticity (HV).

We start with a two-component CQ model in a general rescaled form, which describes spatial evolution of the light beams along the propagation coordinate $z$ in a bulk medium [21],

$$
\begin{aligned}
& i \partial_{z} E_{1,2}+\Delta E_{1,2}+\left(\left|E_{1,2}\right|^{2}+\alpha\left|E_{2,1}\right|^{2}\right) E_{1,2}-\gamma\left(\left|E_{1,2}\right|^{4}\right. \\
& \left.\quad+2 \beta\left|E_{1}\right|^{2}\left|E_{2}\right|^{2}+\beta\left|E_{2,1}\right|^{4}\right) E_{1,2}=0,
\end{aligned}
$$

where $E_{1,2}(x, y, z)$ are the local slowly varying amplitudes of the two waves and the Laplacian $\Delta$ is the diffraction operator acting on the transverse coordinates $(x, y)$. The real parameters $\alpha$ and $\beta$ are the cubic and quintic XPM coefficients, respectively. The quintic coefficient $\gamma$ of the self-phasemodulation (SPM) may be normalized to be 1 (see, e.g., Ref. [22]), which we assume below.

The CQ nonlinearity was experimentally observed in isotropic media, such as glasses [23] and some organic materials [24]. These media also feature nonlinear loss, but the consideration of the conservative model is justified, as the characteristic soliton's length can be made essentially smaller than the absorption length, or the loss may be compensated by gain. In that case, $E_{1}$ and $E_{2}$ may be realized as orthogonally polarized waves, with $\alpha=2 / 3$ for linear and $\alpha$ $=2$ for circular polarizations. The latter case pertains as well to a set of two waves with different carrier wavelengths. Besides that, the model (1) may serve as a rough isotropic approximation for the description of photorefractive media in the low-saturation regime, which is characterized by equal strengths of the XPM and SPM nonlinearities, $\alpha=\beta=1$ [2]. Thus, different values of $\alpha$ and $\beta$ are physically relevant. The estimation of the typical parameters, includingthe transverse size and minimal energy for the generation of vortex solitons, has been recently presented in Ref. [16].

\section{STATIONARY SOLUTIONS AND STABILITY ANALYSIS}

In this work we focus on the vectorial vortex solitons of the $(m, \pm m)$ types with symmetric components:
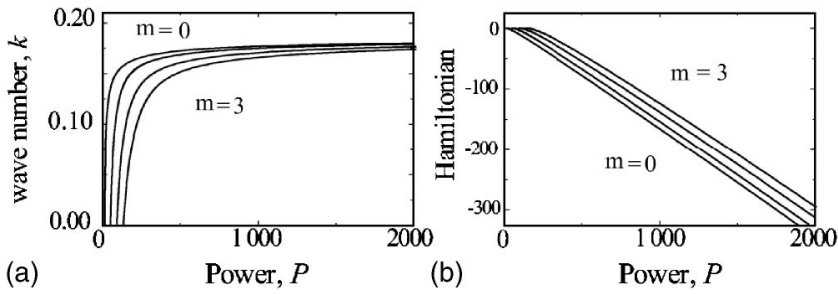

FIG. 1. The wave number $k$ (a) and Hamiltonian (b), calculated as per Eq. (8), versus total power $P=P_{1}+P_{2}$ for the vectorial vortex solitons of the $(m, \pm m)$ types.

$$
\left(\begin{array}{l}
E_{1} \\
E_{2}
\end{array}\right)=V(r) \exp (i k z)\left(\begin{array}{l}
\exp (i m \varphi) \\
\exp ( \pm i m \varphi)
\end{array}\right)
$$

where $r$ and $\varphi$ are the polar coordinates in the plane $(x, y), k$ is a wave number, and the real function $V$ obeys the equation

$$
k V=\hat{D}_{m} V+(1+\alpha) V^{3}-(1+3 \beta) V^{5},
$$

where $\hat{D}_{m} \equiv d^{2} / d r^{2}+r^{-1} d / d r-m^{2} r^{-2}$. Using the transformation $V=R(1+\alpha)^{1 / 2}(1+3 \beta)^{-1 / 2}, r \rightarrow r(1+\alpha)^{-1}(1+3 \beta)^{1 / 2}$, and $k \rightarrow k(1+\alpha)^{-2}(1+3 \beta)$, we cast Eq. (3) in the form

$$
k R=\hat{D}_{m} R+R^{3}-R^{5},
$$

which is supplemented by the boundary condition $R \sim r^{|m|}$ at $r \rightarrow 0$. For $r \rightarrow \infty$, there are two types of solutions to Eq. (4), coexisting in the medium with competing nonlinearities [25] - viz., bright solitons with $R \sim \exp (-\sqrt{k r} r) / \sqrt{r}$ and dark solitons with $R^{2}(r=\infty)=(1+\sqrt{1-4 k}) / 2$. Integral characteristics of the bright vectorial soliton are represented by its partial powers in both components:

$$
P_{1,2} \equiv \frac{2 \pi}{1+\alpha} \int_{0}^{\infty} r d r R^{2}(r)
$$

Global characteristics of the soliton families, in the form of dependences $k(P)$, where the total power is $P \equiv P_{1}+P_{2}$, are displayed in Fig. 1(a) for $\alpha=\beta=1$. The cutoff (largest possible) value of $k$ for the bright-soliton family is the same as for the family of commonly known 1D solitons in the CQ model, which is $k(P=\infty)=3 / 16 \equiv 0.1875$; at this value of $k$, the bright solitons become infinitely broad (approaching a finite maximum amplitude, $R_{\max }=\sqrt{3} / 2$ ) -i.e., they go over into dark solitons. We note that the Vakhitov-Kolokolov criterion $d k / d P>0$, which is a necessary condition for the stability of the solitons [26], is satisfied for all these solutions. Actually, it guarantees stability against radial perturbations, but not against symmetry-breaking ones, which are known to be the source of instability of vortex solitons [3].

Note that the partial angular momenta of the two components are not conserved independently, the integral of motion being only the total angular momentum $M=M_{1}(z)+M_{2}(z)$, where the partial momenta are defined as 


$$
M_{1,2}=\int \operatorname{Im}\left(E_{1,2}^{*} \frac{\partial E_{1,2}}{\partial \varphi}\right) d \mathbf{r}
$$

(recall $\varphi$ is the angular coordinate). Two distinct values of the total angular momentum, $M=2 m P$ and $M=0$, correspond to two types of solutions, $(m, m)$ and $(m,-m)$. Obviously, both solitons coincide in shape; therefore, the diagrams for them shown in Fig. 1 are identical. At the same time, the stability of the two types of the solutions may be completely different. An attempt to describe such a difference, based on the "thin-ring" approximation, was made in a saturable model [14]. Recent results on the calculation of a maximal growth rate of instability in the same model [17] demonstrated the difference of two types of solutions and partial suppression of instability for counterrotating vortices $(m$, $-m)$; however, no stable solitons were found in that case.

A detailed consideration demonstrates that basic stability properties of the vectorial solitons are adequately represented by the case of $\alpha=\beta=1$, on which we focus below. In this case, the Hamiltonian of Eq. (1) is

$$
H=\int\left(\left|\nabla E_{1}\right|^{2}+\left|\nabla E_{2}\right|^{2}-\frac{1}{2} I^{2}+\frac{1}{3} I^{3}\right) d \mathbf{r},
$$

with the total intensity $I=\left|E_{1}\right|^{2}+\left|E_{2}\right|^{2}$. The transformation of variables which leads to the normalized equation (4) amounts to $V(r) \equiv R(r) / \sqrt{2}$, so that $I=R^{2}$. With the latter substitution, Eq. (7) reduces to the Hamiltonian of a scalar vortex soliton with charge $m$ :

$$
H=2 \pi \int_{0}^{\infty}\left[\left(\frac{d R}{d r}\right)^{2}+\frac{m^{2}}{r^{2}} R^{2}-\frac{1}{2} R^{4}+\frac{1}{3} R^{6}\right] r d r .
$$

Perturbed vortex-soliton solutions are sought for in the form [cf. Eq. (2) for the unperturbed ones]

$$
\begin{aligned}
& E_{1}=\exp (i k z+i m \varphi)\left[R(r) / \sqrt{2}+\tilde{f}+\widetilde{g}^{*}\right], \\
& E_{2}=\exp (i k z \pm i m \varphi)\left[R(r) / \sqrt{2}+\widetilde{p}+\widetilde{q}^{*}\right],
\end{aligned}
$$

where $\{\tilde{f}, \widetilde{g}, \widetilde{p}, \widetilde{q}\} \equiv\{f(r), g(r), p(r), q(r)\} \exp \left(\lambda_{s} z+i s \varphi\right)$ with a complex eigenvalue $\lambda_{s}$ and an arbitrary integer azimuthal index $s$. Substitution of these expressions into the linearized equation (1) yields a system

$$
i \lambda_{s}\left(\begin{array}{c}
f \\
g \\
p \\
q
\end{array}\right)=\left[\begin{array}{cccc}
\hat{L}^{+} & A & A & A \\
-A & -\hat{L}^{-} & -A & -A \\
A & A & \hat{L}^{ \pm} & A \\
-A & -A & -A & -\hat{L}^{\mp}
\end{array}\right]\left(\begin{array}{l}
f \\
g \\
p \\
q
\end{array}\right),
$$

where $\hat{L}^{ \pm} \equiv \hat{D}_{m \pm s}-k+R^{2}\left(3 / 2-2 R^{2}\right)$ and $A \equiv R^{2}\left(1 / 2-R^{2}\right)$. The \pm signs in Eq. (10) correspond to the two states $(m, \pm m)$.

Note that in the case of the $(m, m)$ solutions, the matrix in Eqs. (10) has a block $[2 \times 2]$ structure; hence, the eigenmodes degenerate $(f=p$ and $g=q)$ and the linear stability problem reduces to one for the scalar vortex soliton (cf. Refs. $[3,7])$. Therefore, the stability properties of the $(m, m)$-symmetric vectorial vortices are completely identical

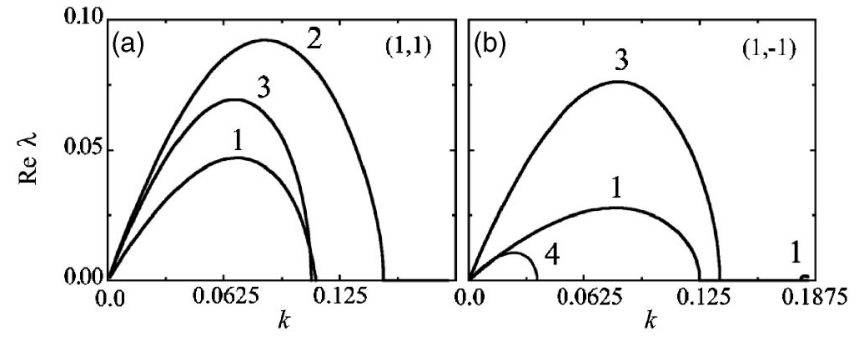

FIG. 2. Growth rates of perturbation eigenmodes with different values of the azimuthal index $s$ (indicated next to the curves) for the vectorial vortex solitons of the types $(1, \pm 1)$.

to those of their scalar counterparts. However, degeneracy does not take place for the HV solitons of the $(m,-m)$ type, which clearly shows the difference in the stability problem for the two types of vectorial vortex solitons.

Stability eigenvalues were found from numerical solution of Eqs. (10). In Fig. 2, we display the dependences of the eigenvalues with different values of the azimuthal index $s$ on the wave number $k$ for vectorial vortex solitons of the $(1,1)$ and $(1,-1)$ types. The maximum growth rate is found for the modes with, respectively, $s=2$ and $s=3$.

The above results comply with the direct simulations of the evolution of the vortices shown in Fig. 3 for $k=0.1$, when the linear stability analysis predicts that the solitons of both types are unstable. The symmetry-breaking instability modes of the $(1,1)$ soliton in both components are identical; therefore, in the Fig. 3(a) we display only one of them. The observed dynamics of two splinters (which are zero-vorticity vector solitons), generated from this solution, is exactly the same as was observed for the scalar vortex soliton: the splinters fly away in tangential directions $[4,7]$.

(a)

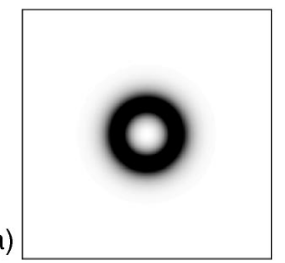
$z=0$
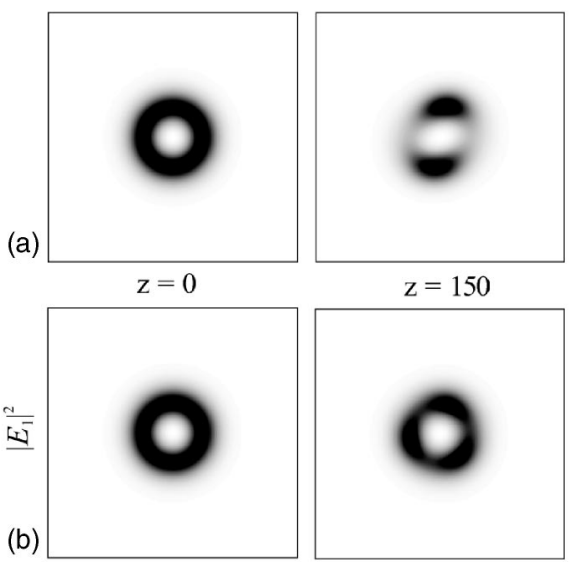

$z=150$
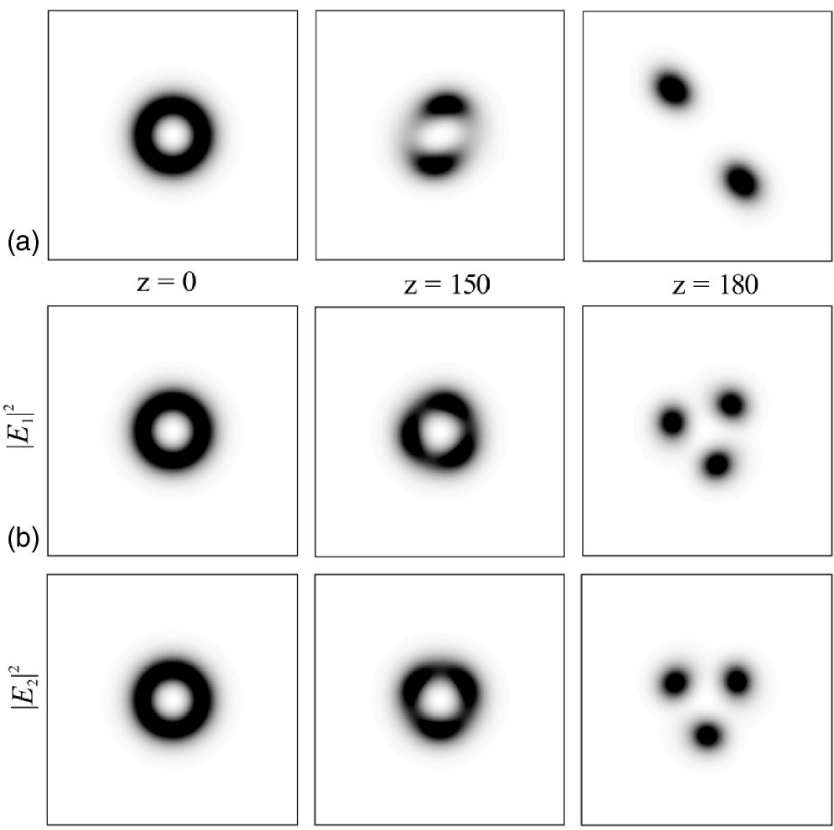

FIG. 3. The instability-induced evolution of the vectorial vortex solitons with $k=0.1$. (a) One of two identical components of the $(+1,+1)$ soliton. (b) Two components of the $(+1,-1)$ soliton with implicit vorticity. 


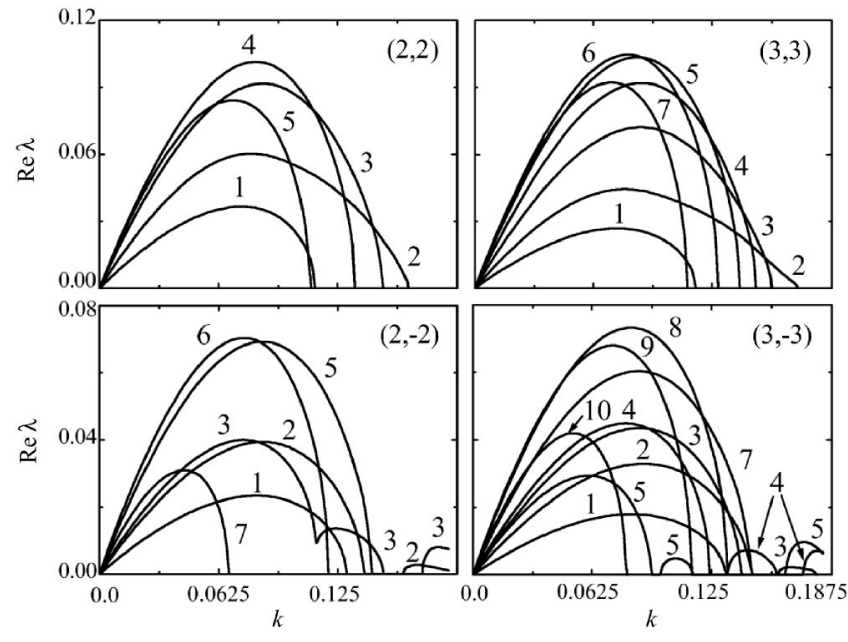

FIG. 4. The same as in Fig. 2 for vectorial vortex solitons of the types $(m, \pm m)$ for $m=2,3$.

A totally different scenario is observed in Fig. 3(b), where, in each component, three splinters of the initial HV soliton start to move in radial directions (cf. similar observations in Refs. [11,27]). At this stage of the HV soliton's breakup, as is seen in the panel corresponding to $z=180$ in Fig. 3(b), the triangular sets of splinters in the two components are slightly misaligned. With further propagation, the separation of the splinters ceases and they eventually fuse into a spinless $(0,0)$ vectorial soliton.

Figure 4 displays the instability growth rates found from Eq. (10) for higher-order vortex solitons, of the $(m, \pm m)$ types, with $m=2$ and 3 . As seen in these figures, each type of the soliton has its stability area, as summarized in Table I. From these results, we conclude that, for all the solitons with explicit vorticity [the $(m, m)$ type], the stability regions extend up to the cutoff value $k=0.1875$, which implies that these solitons continuously carry over into stable vortices of the dark-soliton type, similar to what is known in the scalar case. On the contrary, for the HV solitons [the $(m,-m)$ type], the stability interval never reaches the cutoff value; i.e., dark vortices of the same type are always unstable. In fact, stability intervals for vortex solitons terminating at $k$ smaller than the cutoff value ("stability islands") have never been reported before. In the case of the higher-order solitons (with $m=2$ and 3$)$, the $(m,-m) \mathrm{HV}$ solitons have a smaller stability domain than their $(m, m)$ counterparts. It is noteworthy too

TABLE I. Stability intervals and their size relative to the overall existence interval $0<k<3 / 16 \equiv 0.1875$ for the vectorial vortex solitons of sundry types.

\begin{tabular}{lccr}
\hline \hline$(m, n)$ & Unstable & Stable & $\% \%$ \\
\hline$(1,1)$ & $0<k<0.14855$ & $0.14855<k<0.18750$ & $20.8 \%$ \\
$(2,2)$ & $0<k<0.16190$ & $0.16190<k<0.18750$ & $13.7 \%$ \\
$(3,3)$ & $0<k<0.17005$ & $0.17005<k<0.18750$ & $9.3 \%$ \\
$(1,-1)$ & $0<k<0.13582$ & $0.16163<k<0.17945$ & $9.5 \%$ \\
$(2,-2)$ & $0<k<0.14884$ & $0.15620<k<0.15940$ & $1.7 \%$ \\
$(3,-3)$ & $0<k<0.15866$ & $0.15866<k<0.15973$ & $0.57 \%$ \\
\hline \hline
\end{tabular}

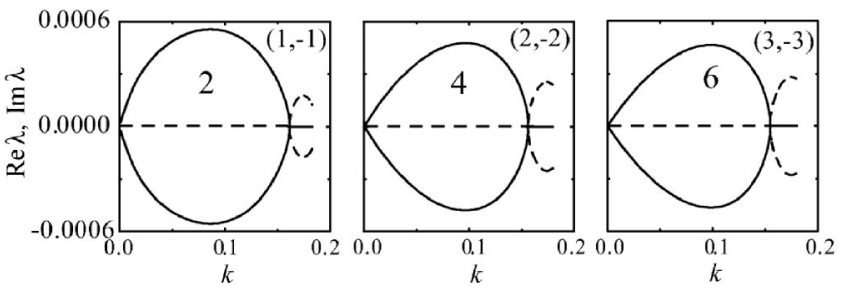

FIG. 5. Real (solid lines) and imaginary (dashed lines) parts of the instability eigenvalues of the small "internal" instability eigenmodes for the hidden-vorticity solitons of the type $(m,-m)$ with $m=1,2$, and 3 , the corresponding azimuthal indices being $s=2 m$.

that the azimuthal index $s$ of the most unstable eigenmode depends on the type of the soliton, and for the higher-order ones of the types $(2, \pm 2)$ and $(3, \pm 3)$, the most dangerous value of $s$ depends on $k$ as well.

\section{CHARGE FLIPPING}

Performing a numerical analysis of the linear-stability problem based on Eq. (10) with higher accuracy, we have found additional very small unstable eigenvalues for even azimuthal indices $(s=2 m)$, with the growth rate $\simeq 5 \times 10^{-4}$, which were not visible in Figs. 2 and 4. In Fig. 5 we plot corresponding ordinary bifurcations of the pitchfork type. In order to check the numerical accuracy we applied two different discretization meshes and found that the numerical values of these minuscule eigenvalues coincide within the first two significant digits when passing from a grid with 300 points to a grid with 600 points. As a result of these additional very weak perturbations, the corresponding rigorously defined stability regions for the $(m,-m) \mathrm{HV}$ solitons are considerably reduced with respect to their $(m, m)$ counterparts (see Table I for a summary of the output of the stability calculations).

To visualize the development of the weak instability revealed by Fig. 5, we simulated the propagation of the solitons of both types $(1, \pm 1)$ with $k=0.14$, adding random noise with a relative amplitude of $10 \%$. For the solitons with the explicit vorticity, the instability growth rate is $\operatorname{Re} \lambda=0.0373$, and the dynamics follows the "usual" break-up scenario shown in Fig. 3(a); therefore, we do not display it again. The growth rate of the same instability mode with $s=2$ but for the HV soliton of the $(1,-1)$ type is two orders of magnitude smaller, $\operatorname{Re} \lambda \approx 0.00036$; therefore, noticeable development of the instability should be expected after having passed the distance $\sim 10^{4}$. Although the so large propagation distances can hardly be achieved experimentally (in the experiment, these solitons will seem as completely stable ones), the issue is of principal interest; therefore, we have completed the numerical analysis and found the soliton's dynamics of a novel kind. The results are summarized in Fig. 6, where we show the intensity and phase distributions for both components up to $z=175000$.

To explain the complex internal dynamics observed in Fig. 6, first we recall the theory developed for necklace-ring vector solitons in Ref. [11]. The HV solutions that we consider here may be regarded as constituting a special type of 

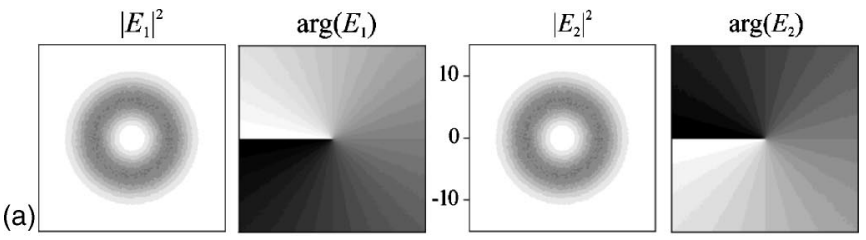

(b)
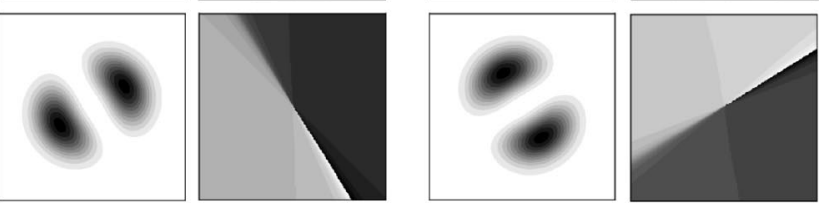

(c)
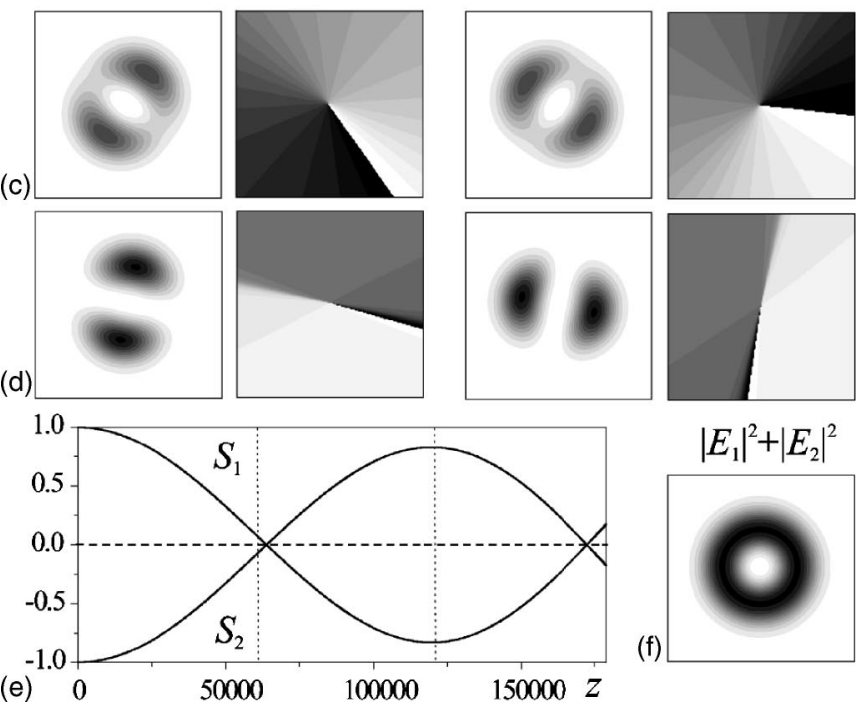

FIG. 6. Evolution of the "internally unstable" vortex soliton with the hidden vorticity, of the $(1,-1)$ type, for $k=0.14$. The propagation distance is $z=0$ (a), 60000 (b), 120000 (c), and 175000 (d). The dynamical exchange of the spins $S_{1,2}$ [solid lines in (e)] is accompanied by charge flipping (sign reversal of the vorticity in each component), as is clearly visible in the phase diagrams for $\arg \left(E_{1,2}\right)$ in (a)-(d). The total spin $S$ keeps its zero value [see the dashed line in (e)]. The net intensity $\left|E_{1}\right|^{2}+\left|E_{2}\right|^{2}$ keeps its azimuthal homogeneity in the course of the entire propagation, as shown in (f) for $z=175000$. Vertical dotted lines correspond to snapshots (b) and (c).

the necklace-ring vectorial solitons, with equal powers in both components, $P_{1,2}=P / 2$. In the most general case, these solutions may be represented as [cf. Eq. (2)]

$$
\left(\begin{array}{c}
E_{1} \\
E_{2}
\end{array}\right)=R(r) e^{i k z} \boldsymbol{\Theta} \Psi \boldsymbol{\Phi}\left(\begin{array}{c}
\cos (m \varphi) \\
\sin (m \varphi)
\end{array}\right) .
$$

Here the matrix

$$
\boldsymbol{\Theta}=\left[\begin{array}{cc}
e^{i \theta_{1}} & 0 \\
0 & e^{i \theta_{2}}
\end{array}\right],
$$

with arbitrary constants $\theta_{1,2}$, indicates the phase invariance of the solutions; this symmetry property is amenable for the conservation of the partial powers $P_{1,2}$. The matrix $\boldsymbol{\Phi}$ is simply a rotational transformation in the transverse plane,

$$
\boldsymbol{\Phi}=\left[\begin{array}{cc}
\cos \left(m \varphi_{0}\right) & \sin \left(m \varphi_{0}\right) \\
-\sin \left(m \varphi_{0}\right) & \cos \left(m \varphi_{0}\right)
\end{array}\right],
$$

where the arbitrary constant angle $\varphi_{0}$ reflects the rotational invariance responsible for the conservation of the total angular momentum. Note that, for radially symmetric solutions of Eq. (2), the transformation $\varphi \rightarrow \varphi_{0}$, described by Eq. (13), is equivalent to the phase shift accounted for by Eq. (12) with $\theta_{1,2}=\mp m \varphi_{0}$.

The arbitrary phase shift being already absorbed by the matrix $\boldsymbol{\Theta}$, the linear transformation $\boldsymbol{\Psi}$ in Eq. (11) is given by

$$
\boldsymbol{\Psi}=\left[\begin{array}{cc}
\cos \psi & i \sin \psi \\
\sin \psi & \pm i \cos \psi
\end{array}\right]
$$

where the constant parameter $\psi$ describes the rotation in the space of the components $\left(E_{1}, E_{2}\right)$, similar to the Manakov system, and corresponds to conservation of the "isotopic spin," $i \int\left\{E_{1} E_{2}^{*}-E_{1}^{*} E_{2}\right\} d \mathbf{r}$. For any value of $\psi$, the latter expression is zero in our case. In addition, the parameter $\psi$ uniquely defines the initial values of the partial spins $S_{n}$ $=M_{n} / P_{n}$ [recall the partial angular momenta were defined in Eq. (6)],

$$
S_{1}=m \sin (2 \psi), \quad S_{2}= \pm S_{1},
$$

and, therefore, it determines the total spin (dynamical invariant) $S \equiv M / P=\frac{1}{2}\left(S_{1}+S_{2}\right)=\frac{1}{2}(m \pm m) \sin (2 \psi)$.

Among possible stationary solutions conforming to Eq. (11) are those with zero, fractional $(0<S<m)$, and integer total spin $(S=m)$. We focus here on two cases which correspond to Eq. (2): the $(m, m)$ type of the solutions, with the total angular momentum attaining its maximum possible value, $M=m P$ (i.e., $S=m)$, and the HV solutions of $(m$, $-m$ ) type, with $M=S=0$. Both of them represent radially symmetric vector vortices with $|\psi|=\pi / 4$; the transformations $\psi \rightarrow-\psi, m \rightarrow-m$, and $(m, \pm m) \rightarrow(-m, \mp m)$ are all equivalent.

Solutions with explicit vorticity $S=m$ correspond to the upper sign in Eq. (14). The partial spins assume the maximum possible values in this case, $S_{1,2}=m$; thus, the only corresponding configuration is the one with $|\psi| \equiv \pi / 4$, and the vortex soliton of this type always has axially symmetric (ring-shaped) components. In addition, the exchange of angular momentum between components is forbidden in this case.

The HV solutions with $S=0$ are drastically different. They correspond to the lower sign in Eq. (14) for an arbitrary value of $\psi$. These include the $\mathrm{HV}$ vectorial vortices for $\psi$ $= \pm \pi / 4$ and also solutions with intensity distributions in the two components in the form of two crossed multipoles, for $\psi=0$ and $S_{1,2}=0$. A similar solution with $m=1$, or a dipoledipole vectorial soliton, was investigated theoretically and experimentally in Refs. [11,27] and found to be azimuthally unstable in a saturable medium. The $\psi$ values from the interval $-\pi / 4<\psi<\pi / 4$ determine the depth of the azimuthal modulation in each of the two components, which sum up to 


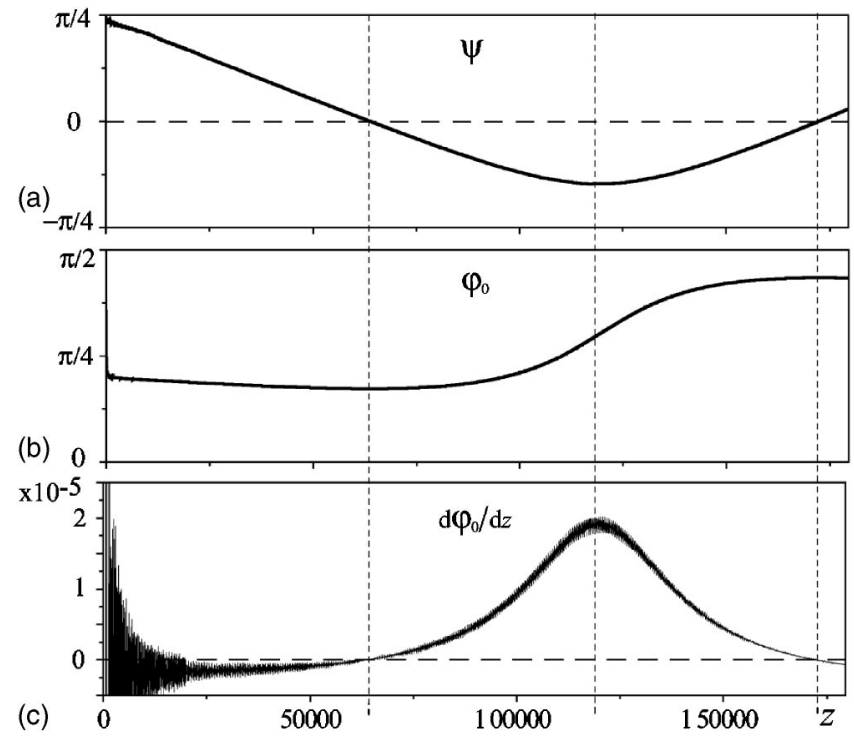

FIG. 7. Evolution of the parameters $\psi$ from Eq. (14) in (a) and $\varphi_{0}$ from Eq. (13) in (b), corresponding to the adiabatic "sliding" across the soliton family, defined by Eq. (11) in the course of the evolution displayed in Fig. 6. In (c), the angular velocity of the internal rotation is shown; strong numerical noise on this curve reflects the uncertainty of the value of the parameter $\varphi_{0}$ which is arbitrary for the unperturbed stationary solutions. Vertical dashed lines indicate the charge-flipping (spin-reversal) points, close to those shown in Figs. 6(b) and 6(d).

the azimuthally uniform distribution of the total intensity $I$ $=R^{2}$ [11]. Thus, the continuous soliton family includes crossed multipoles with different values of the azimuthal modulation depth and opposite fractional values of the partial spins. It is important to note that, because all the dynamical invariants do not depend on $\psi$, for the whole branch of HV vectorial solitons parametrized by $\psi$, dynamical exchange of angular momentum between components is possible.

Using the decomposition in Eq. (11), we can now explain the dynamics in Fig. 6 in terms of a slow evolution of the parameters $\varphi_{0}$ and $\psi$, as shown in Fig. 7. In other words, instead of modulational instability along the azimuthal direction, which would lead to fragmentation of the initial annular soliton, the instability modes from Fig. 5 initiate slow sliding of the solution across the continuous manifold with the independent parameters $\varphi_{0}$ and $\psi$. At each stage of the evolution, such as those corresponding to frames (a)-(d) in Fig. 6, we observe a slightly perturbed stationary solution with varying $\varphi_{0}$ and $\psi$ (the arbitrary phases $\theta_{1,2}$ are of no importance because the XPM interaction between the components is phase insensitive).

Indeed, the random noise at the level of $10 \%$ of the soliton's amplitude, added to the HV soliton in Fig. 6(a), quickly dissipates, and in the course of the first several thousands units, the vector HV soliton propagates without any noticeable change. Then, as is seen in Fig. 7(a), the parameter $\psi$ decreases and the components assume a shape of two crossed dipoles. When the modulation depth reaches its maximum for $\psi=0$, the solution is, simply, $\left\{E_{1}, E_{2}\right\}=R(r)\{\cos (\varphi$ $\left.\left.-\varphi_{0}\right), \sin \left(\varphi-\varphi_{0}\right)\right\}$; hence, at this point, neither component contains any vorticity, as is indeed seen in Fig. 6(b). Because the parameter $\varphi_{0}$ is arbitrary, its particular value at this stage [Fig. 7(b)] depends on the initial noise.

With further propagation, the components $E_{1,2}$ almost restore their initial annular shapes and develop phase dislocations corresponding to new vortex cores, which are opposite to initial ones [cf. Figs. 6(a) and 6(c)]. This phenomenon, "charge flipping," was recently predicted to occur in a significantly different system: namely, vortices in nonlinear photonic lattices [28]. The latter system does not conserve the angular momentum at all, because the rotational symmetry is broken by the lattice. Nevertheless, the similarity with that system, which seems to be important for the effect to occur, is the presence of two interacting subsystems which are given the freedom to exchange the angular momentum: the vortex and the lattice in Ref. [28] and the two components of the vortex soliton in the present case. It is commonly known from the studies of vortices in linear optics [29] that vortices in the phase fronts can annihilate or be born in pairs. The charge-flipping phenomenon introduces a new mechanism of such transformation in the nonlinear setting, through the exchange of the angular momentum between two nonlinearly coupled subsystems.

After the first charge flip, the components do not fully restore their annular shape [Fig. 6(c)], and the maximum value of the partial spins which have the opposite signs, $S_{2}$ $=-S_{1} \approx 0.83$ with $\psi \approx-0.462$, is attained at $z \approx 117300$ [Fig. 7(a)]. At the same time, the dipoles in both components start to rotate slowly [the angle $\varphi_{0}$ increases; see Fig. 7(b)]. We can introduce, therefore, the angular velocity of the rotation, $d \varphi_{0} / d z$, as shown in Fig. 7(c). However small, it demonstrates an important feature of the correlation between the internal "degrees of freedom" $\psi$ and $\varphi_{0}$. Indeed, for the exact stationary solutions, these two parameters are independent, while for the perturbed solutions in Figs. 6 and 7, they become coupled through the growing instability modes. In particular, points where the angular velocity vanishes correspond to a dipole-dipole soliton with zero vorticity in both components, while the fastest rotation is achieved when the partial spins in the components attain maximum absolute values.

We continued the simulations and eventually observed a breakup of the vortex soliton, as shown in Fig. 8. It occurs within the distance of several hundreds of propagation units-i.e., three orders of magnitude smaller than the previous stable propagation; thus, it may be regarded as an "explosion." We have checked the evolution of the integrals of motions at this stage and, in particular, observed perfect conservation of the total spin, Fig. 8(d), which rules out a numerical error as a probable cause of the "explosion."

In Fig. 9 we show the total integral power and Hamiltonian, calculated in the course of the propagation, and notice small changes which naturally occur due to the radiation emission from the perturbed soliton. The breakup of the soliton is accompanied by a strong emission of radiation which leads to sharp changes in the final segment of the diagrams. Using the relations displayed in Fig. 1, we restore the corresponding value of the propagation constant $k$ and plot it in Fig. 9(c). As one can see, the propagation constant decreases, parallel to the power loss due to the radiation. The explosion 

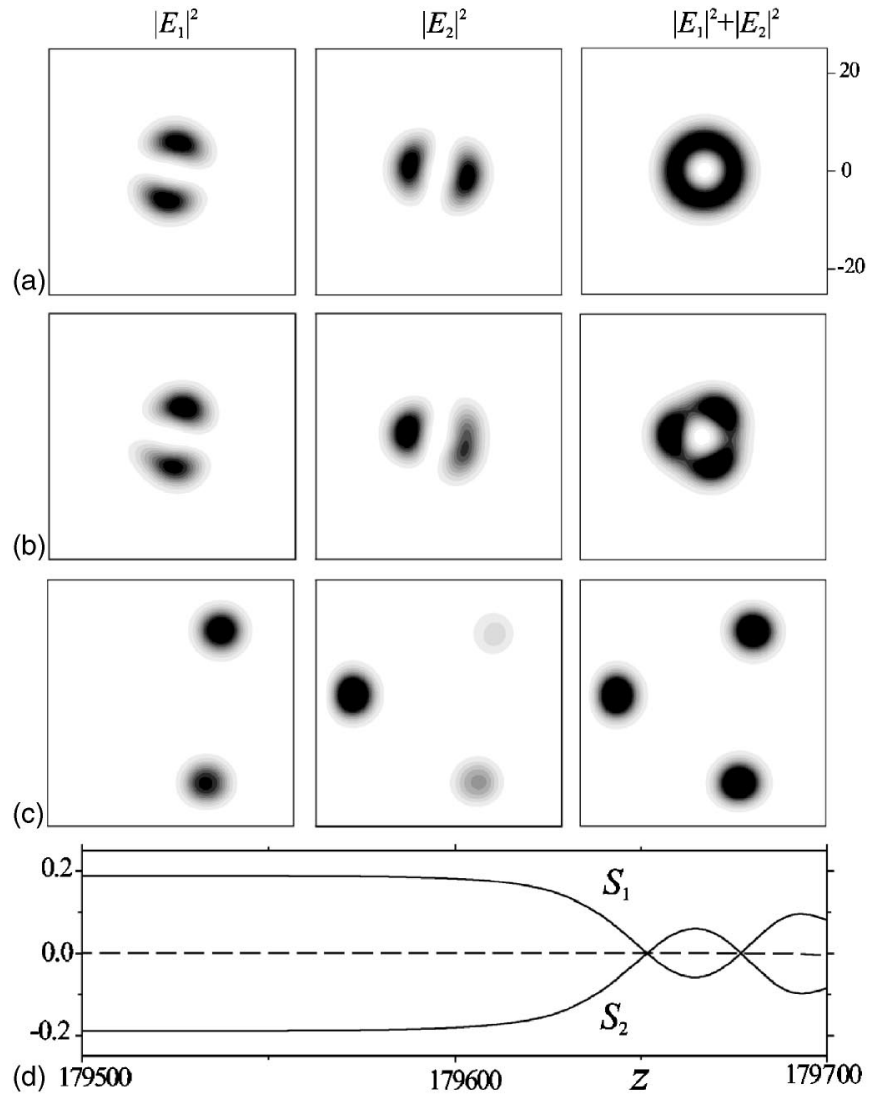

FIG. 8. The final stage of the soliton's evolution, featuring the "explosion," after the quasistable evolution in Fig. 6. The propagation distance is $z=179500$ in (a), 179600 in (b), and 179700 in (c).

occurs when the propagation constant reaches the value $k$ $\approx 0.13627$, and the splitting in Fig. 8 follows the scenario already observed in Fig. 3-three splinters fly away along radial directions. We conclude that the sudden splitting of the otherwise "intrinsically" unstable HV soliton happens be-

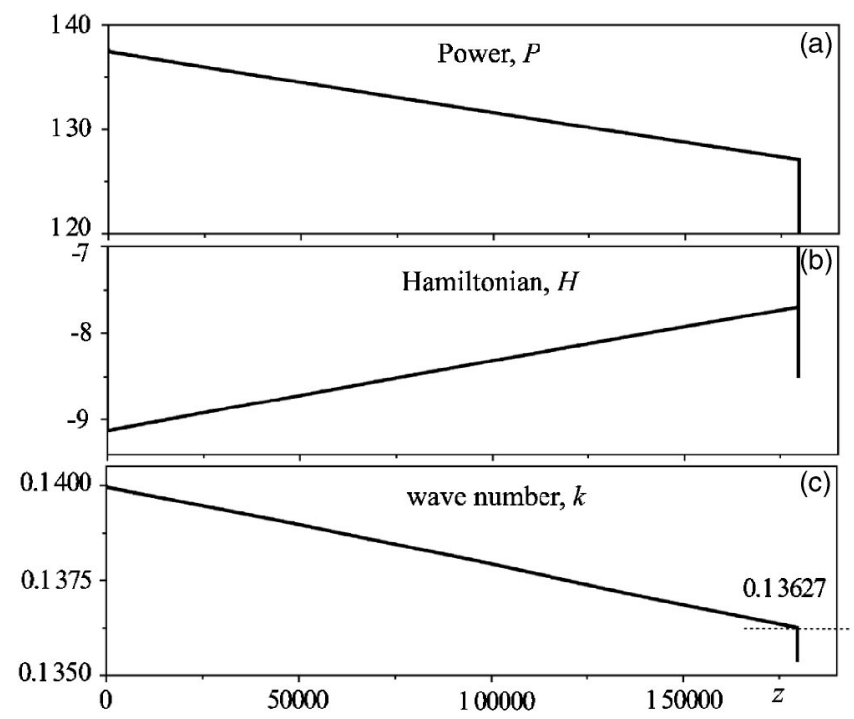

FIG. 9. Evolution of the total integral power (a), Hamiltonian (b), and accordingly defined propagation constant (c) in the course of the propagation shown in Figs. 6 and 8. cause it hits a boundary of the "external" instability domain, after which it breaks apart. The latter boundary is found from the linear-stability analysis to be at $k=0.13582$ (see Table I), and we stress the remarkable agreement and accuracy of the numerical procedure: both values coincide up to $10^{-3}$-i.e., $k \approx 0.136$.

Closer to the linear-stability domain-for instance, for $k$ $=0.16$ - the HV soliton of the type $(1,-1)$ demonstrates no sign of instability for any reasonable propagation distance despite the addition of initial noise, because the corresponding instability mode with $s=2$ in Fig. 3 has a vanishingly small growth rate. We mark the vanishing "internal" instability by excluding the corresponding domain from the final summary in Table I.

We conjecture that the internal dynamics of the vectorial soliton and charge flipping reported above for the particular CQ model and the particular case of $m=1$ may manifest itself for higher topological charges as well as in other systems-for example, in the mixture of Bose-Einstein condensates (BEC's) [30]. In the latter system, the overall stabilization of vortices is provided by the external trap, rather than the quintic nonlinearity. The necklace-ring-type solutions in two-component BEC's, or nontopological vortices, were recently described in Ref. [31]. It is interesting to note that the internal dynamics of vector vortices, which keeps the overall density profile intact similar to Fig. 6, can be initiated in a BEC mixture by the adiabatic or abrupt changes of the external coupling drive; however, no charge-flipping effect was observed in Ref. [31].

\section{INSTABILITY OF THE VORTEX CORE CLOSE TO CUTOFF}

As was already stressed, Figs. 2 and 4 emonstrate that, in contrast to the stability domain for the solitons of the $(m, m)$ type, the stability region for the HV solitons of the $(m,-m)$ type does not extend to the cutoff point. In this section, we aim to study the instability of the HV solitons close to this point. The simulations demonstrate that the corresponding instability mode with the azimuthal index $s=1$ leads to a shift of the vortex core. A possibility of this specific instability was earlier studied in Ref. [32] for vortices in scalar models, using an analytical approximation for very broad annular solitons. It was concluded that such an instability may occur, but no particular linear-instability mode corresponding to the core shift was found [as before, the scalar case is exactly tantamount to the symmetric vectorial vortices of $(m, m)$ type considered here]. In contrast to that, Fig. 10 shows an explicit example of such an instability mode for the HV solitons of the $(1,-1)$ type.

Numerical development of this instability is displayed for $k=0.18$ in Fig. 11. Adding initial random noise with a $20 \%$ relative amplitude [Fig. 11(a)] does not strongly affect the dynamics - for up to 4000 propagation units, it shows no sign of instability. The only visible action of the perturbation is excitation of internal modes of the vectorial soliton, corresponding to purely imaginary eigenvalues in the linearperturbation spectrum [such eigenvalues are shown in Fig. 10(a)]. The pattern displayed in Fig. 11(b) periodically re- 

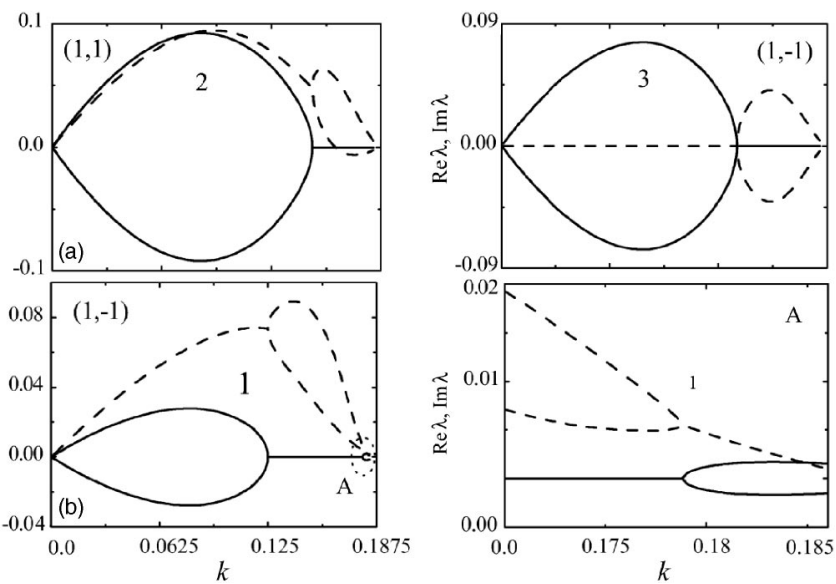

FIG. 10. (a) Real (solid lines) and imaginary (dashed lines) parts of the instability eigenvalues with the maximum growth rate for the vectorial solitons of the $(1, \pm 1)$ types. (b) An additional bifurcation (magnified in the inset A) of the perturbation mode with $s=1$, which occurs for the hidden-vorticity soliton of the $(+1,-1)$ type close to the cutoff.

peats itself during the propagation. Similar long-lived internal modes have been recently observed in perturbed evolution of scalar CQ vortex solitons [33]. It is noteworthy too that, for the symmetric vectorial vortex soliton shown in Fig. 11, the total intensity shows no sign of a growing azimuthal modulation, as the intensity of the components sum up to the axially uniform distribution, similar to the case of "intrinsic" instability shown in Fig. 6.

At the distance of $z=4300$, the growth of the unstable mode results in separation of the dislocations in the two components [see Fig. 11(c)]. The distribution of the total intensity remains unmodulated, and the vortex core is invisible, because it is covered, in the total-intensity distribution, by the mutual displacement of the components: actually, an intensity maximum in one component lies on top of a minimum in the other. The shift of the vortex core quickly increases, and it moves to the outer edge of the soliton. As a whole, the vectorial soliton remains localized, as is seen in Fig. 11(d), and it possesses no vorticity, as the spin diagrams in Fig. 11(e) demonstrate. Strong modulation of the components inside the soliton persists for a long propagation distance after the vortex annihilation. We conclude that, in this case, the result of the development of the instability mode is establishment of a breather-i.e., a strongly perturbed and oscillating zero-spin soliton.

\section{CONCLUSION}

We have demonstrated that 2D spatial solitons of annular shape carrying zero total vorticity may be stable in the CQ medium, being supported by the hidden (implicit) vorticity, in quite a broad region. The output of the linear-stability analysis is summarized in Table I, where the stability domain and its size relative to the existence domain are shown. There is a single border between instability and stability regions for the explicit-vorticity solitons of the $(m, m)$ type and the stability domain extends up to the point of the transition to

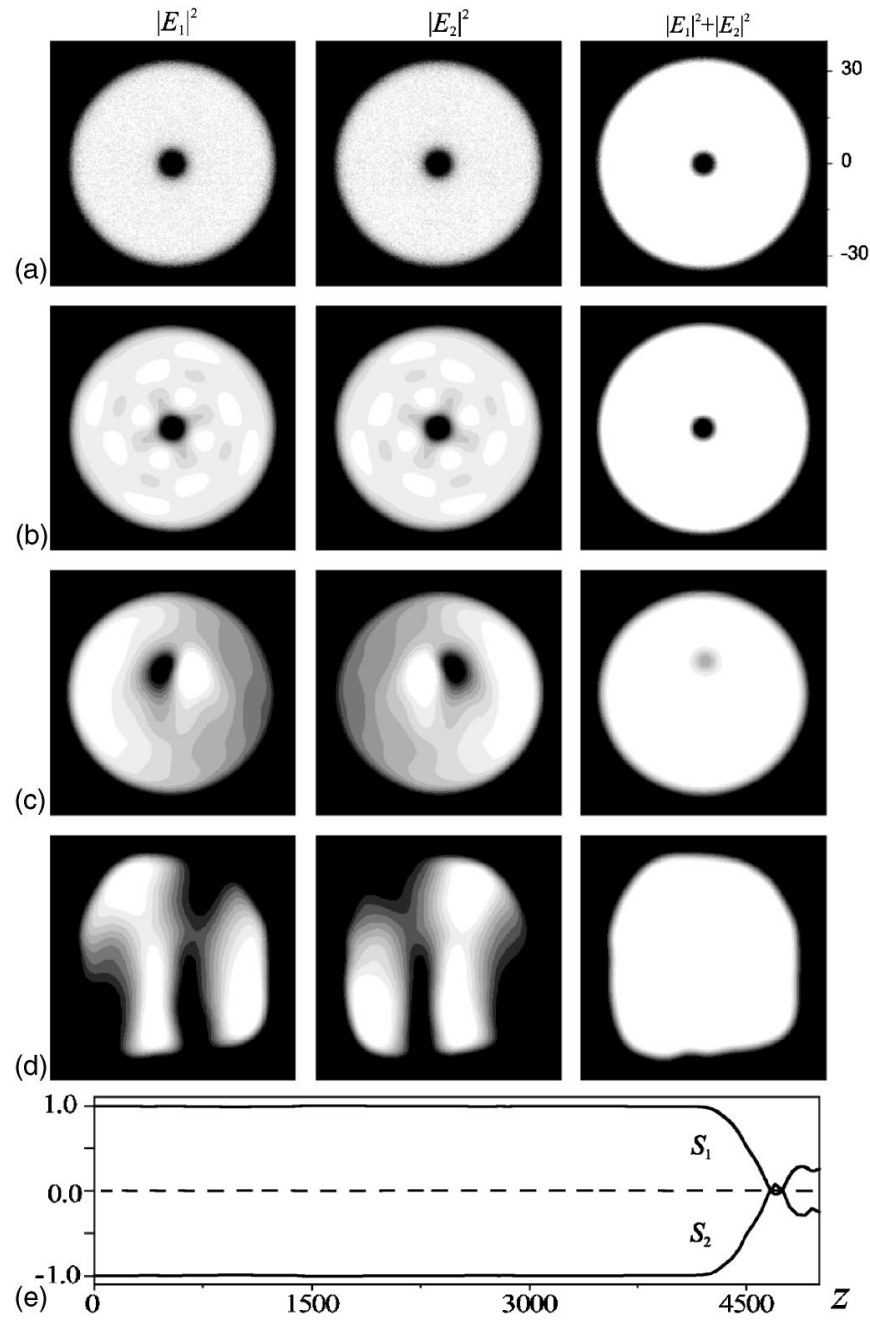

FIG. 11. Dynamics of the hidden-vorticity soliton of the (1, $-1)$ type with $k=0.18$ and initial $20 \%$ random-noise perturbation. The propagation distance is $z=0$ in (a), 500 in (b), 4300 in (c), and 5000 in (d). In this figure, in contrast to previous ones, the intensity is scaled from black (zero) to white (maximum). As before, the frame (e) demonstrates the evolution of the partial spins (solid lines) in the two components and conservation of the total angular momentum (the dashed line).

dark-soliton vortices (cutoff). For the implicit-vorticity solutions of the $(m,-m)$ type, the situation is more complex. We identify the region of the relatively strong "external" instability (shown in Table I), where unstable vectorial solitons with both implicit and explicit vorticity split into a set of fragments, the number of which is equal to the azimuthal index of the fastest growing mode of small perturbations. For larger $k$ and only for the hidden-vorticity solitons of the $(m,-m)$ type, there exists a domain of very weak "intrinsic" instability, where the vectorial soliton as a whole remains robust, while its components undergo a very slow internal evolution, "sliding" through the solution family and exhibiting exchange of the angular momentum and charge flipping. In a vicinity of the cutoff, where the explicit vortices are stable, the hidden-vorticity solitons reveal a weak-instability 
mode that results in splitting of the phase dislocations in the two components, somewhat similar to the splitting of multiple-charged dark vortex solitons in the scalar model. Therefore, the stable solitons with hidden vorticity may be regarded as "exceptionally bright" objects, unlike the familiar solitons carrying explicit vorticity, which always have stable dark-vortex counterparts. With the increase of the integer vorticity $m$, the stability regions of the vectorial solitons with both explicit and implicit vorticity quickly shrink.

\section{ACKNOWLEDGMENTS}

A.S.D. gratefully acknowledges support from the Alexander von Humboldt Foundation. The work of B.A.M. was supported, in a part, by the Israel Science Foundation through Grant No. 8006/03. This author appreciate hospitality of the Nonlinear Physics Centre at the Research School of Physical Sciences and Engineering, Australian National University. D.M. and D.M. acknowledge support from Deutsche Forschungsgemeinschaft (DFG), Bonn.
[1] Yu. S. Kivshar and G. P. Agrawal, Optical Solitons: From Fibers to Photonic Crystals (Academic Press, San Diego, 2003).

[2] For an overview of the field see Solitons, edited by M. Segev[Opt. Photonics News 13, 27 (2002)].

[3] L.-C. Crasovan, B. A. Malomed, and D. Mihalache, Pramana, J. Phys. 57, 1041 (2001).

[4] W. J. Firth and D. V. Skryabin, Phys. Rev. Lett. 79, 2450 (1997); D. V. Skryabin and W. J. Firth, Phys. Rev. E 58, 3916 (1998).

[5] L. Torner and D. V. Petrov, Electron. Lett. 33, 608 (1997); D. V. Petrov, L. Torner, J. Martorell, R. Vilaseca, J. P. Torres, and C. Cojocaru, Opt. Lett. 23, 1444 (1998).

[6] M. Quiroga-Teixeiro and H. Michinel, J. Opt. Soc. Am. B 14, 2004 (1997).

[7] R. L. Pego and H. A. Warchall, J. Nonlinear Sci. 12, 347 (2002).

[8] I. Towers, A. V. Buryak, R. A. Sammut, and B. A. Malomed, Phys. Rev. E 63, 055601(R) (2001); D. Mihalache, D. Mazilu, B. A. Malomed, and F. Lederer, ibid. 69, 066614 (2004).

[9] M. Soljaćić, S. Sears, and M. Segev, Phys. Rev. Lett. 81, 4851 (1998); M. Soljaćić and M. Segev, Phys. Rev. E 62, 2810 (2000); Phys. Rev. Lett. 86, 420 (2001).

[10] A. S. Desyatnikov, D. Neshev, E. A. Ostrovskaya, Yu. S. Kivshar, G. McCarthy, W. Krolikowski, and B. Luther-Davies, Opt. Lett. 26, 435 (2001); J. Opt. Soc. Am. B 19, 586 (2002).

[11] A. S. Desyatnikov and Yu. S. Kivshar, Phys. Rev. Lett. 87, 033901 (2001).

[12] J. J. Garcia-Ripoll, V. M. Perez-Garcia, E. A. Ostrovskaya, and Yu. S. Kivshar, Phys. Rev. Lett. 85, 82 (2000); W. Krolikowski, E. A. Ostrovskaya, C. Weilnau, M. Geisser, G. McCarthy, Yu. S. Kivshar, C. Denz, and B. Luther-Davies, ibid. 85, 1424 (2000); T. Carmon, C. Anastassiou, S. Lan, D. Kip, Z. H. Musslimani, and M. Segev, Opt. Lett. 25, 1113 (2000)

[13] J. Yang and D. E. Pelinovsky, Phys. Rev. E 67, 016608 (2003).

[14] M. S. Bigelow, Q.-H. Park, and R. W. Boyd, Phys. Rev. E 66, 046631 (2002).

[15] D. Mihalache, D. Mazilu, I. Towers, B. A. Malomed, and F.
Lederer, Phys. Rev. E 67, 056608 (2003); J. Opt. A, Pure Appl. Opt. 4, 615 (2002).

[16] D. Mihalache, D. Mazilu, B. A. Malomed, and F. Lederer, J. Opt. B: Quantum Semiclassical Opt. 6, S341 (2004).

[17] F. Ye, J. Wang, L. Dong, and Y. P. Li, Opt. Commun. 230, 219 (2004).

[18] A. V. Mamaev, M. Saffman, and A. A. Zozulya, J. Opt. B: Quantum Semiclassical Opt. 6, S318 (2004).

[19] J. P. Torres, J. M. Soto-Crespo, L. Torner, and D. V. Petrov, Opt. Commun. 149, 77 (1998).

[20] H. Leblond, B. A. Malomed, and D. Mihalache, Phys. Rev. E (to be published).

[21] A. I. Maimistov, B. A. Malomed, and A. Desyatnikov, Phys. Lett. A 254, 179 (1999).

[22] A. Desyatnikov, A. Maimistov, and B. Malomed, Phys. Rev. E 61, 3107 (2000).

[23] F. Smektala, C. Quemard, V. Couderc, and A. Barthélémy, J. Non-Cryst. Solids 274, 232 (2001).

[24] C. Zhan, D. Zhang, D. Zhu, D. Wang, Y. Li, D. Li, Z. Lu, L. Zhao, and Y. Nie, J. Opt. Soc. Am. B 19, 369 (2002).

[25] V. I. Berezhiani, V. Skarka, and N. B. Aleksic, Phys. Rev. E 64, 057601 (2001).

[26] M. G. Vakhitov and A. A. Kolokolov, Izv. Vyssh. Uchebn. Zaved., Radiofiz. 16, 1020 (1973) [Radiophys. Quantum Electron. 16, 783 (1973)].

[27] M. Ahles, K. Motzek, A. Stepken, F. Kaiser, C. Weilnau, and C. Denz, J. Opt. Soc. Am. B 19, 557 (2002).

[28] T. J. Alexander, A. A. Sukhorukov, and Yu. S. Kivshar, Phys. Rev. Lett. 93, 063901 (2004).

[29] M. S. Soskin and M. V. Vasnetsov, in Progress in Optics, edited by E. Wolf (North-Holland, Amsterdam, 2001), Vol. 42, p. 219.

[30] T. J. Alexander, E. A. Ostrovskaya, Yu. S. Kivshar, and P. S. Julienne, J. Opt. B: Quantum Semiclassical Opt. 4, S33 (2002).

[31] Q.-H. Park and J. H. Eberly, Phys. Rev. A 70, 021602(R) (2004).

[32] B. A. Malomed, L.-C. Crasovan, and D. Mihalache, Physica D 161, 187 (2002).

[33] L. Dong, F. Ye, J. Wang, T. Cai, and Y. P. Li, Physica D 194, 219 (2004). 